\title{
Incidence and prevalence of juvenile arthritis in an urban population of southern Germany: a prospective study
}

\author{
St von Koskull, H Truckenbrodt, R Holle, A Hörmann
}

\begin{abstract}
Objective-To ascertain the incidence and prevalence of juvenile arthritis in a German urban population.

Methods-All 766 paediatricians, orthopaedists, and rheumatologists working in practices or outpatient clinics in 12 south German towns were asked to report all patients who consulted them for juvenile arthritis during the year 1995. Patients with continuing symptoms were followed up for 9-12 months to obtain a final diagnosis. Extended measures of quality control were taken to control for known biases.

Results-Of 457 reported cases, 294 were diagnosed with para-/postinfectious arthritis (PPA), 78 with juvenile chronic arthritis (JCA), and 18 with other forms of arthritis. Half of the PPA cases were classified as transient synovitis of the hip (SH). For JCA the reported annual incidence was 6.6 and the prevalence 14.8 per 100000 subjects under 16 years of age. For PPA the reported incidence was 76 and the prevalence 4.4 per 100000 subjects under 16. The incidence of rheumatic fever was clearly below 1 per 100000 people under 16. A correction model was used to control for known biases and to adjust the estimates accordingly.
\end{abstract}

Conclusions-The results of this first prospective study on the incidence and prevalence of juvenile arthritis in Germany are consistent with a retrospective study performed in the Berlin area. Based on these results it was estimated that the annual frequency of juvenile arthritis in Germany is as follows: $750-900$ incident JCA cases, 21000 incident $\mathrm{SH}$ cases, and 21000 incidence cases of other forms of PPA a year. The number of incidence cases of rheumatic fever is expected to be markedly lower than 150 a year. The total prevalence is expected to be $3600-4350$ JCA cases, 2250-3000 SH cases, and the same number of other forms of PPA.

(Ann Rheum Dis 2001;60:940-945)

Reliable information on the incidence and prevalence of arthritis among children and adolescents is essential for effective healthcare management in paediatric rheumatology. These data are also necessary as a baseline in order to detect secular changes in the incidence and prevalence.
Table 1 Entry criteria

- Age $<16$ years

- Permanent residence in study area

- Consultation of one of the study doctors from 1 January to 31 December 1995

- Arthritis suspected or confirmed

- Exclusions: trauma, confirmed septic arthritis, concomittant arthritis in malignant diseases

Before this study was performed, there were no reliable data on incidence and prevalence of juvenile arthritis in Germany and estimates had to be based on studies from other countries. ${ }^{1}$ However, such results cannot simply be transferred to a different region because there may be geographical as well as secular differences in the incidence and prevalence of juvenile arthritis. Several studies have been performed on juvenile chronic arthritis (JCA) in Europe, ${ }^{2-5}$ but epidemiological studies of acute forms of juvenile arthritis are rare. ${ }^{36}$

\section{Methods}

A prospective, population based survey was performed in the study area consisting of 12 towns and cities in Bavaria, including Munich and Nuremberg.

A total of 364939 people under the age of 16 years had their permanent residence in these cities on 31 December 1994. This is approximately $17.4 \%$ of the Bavarian and $2.6 \%$ of the German population of that age group. We identified all paediatricians, orthopaedists, and rheumatologists $(n=766)$ of these 12 towns and several selected neighbouring suburbs. All these doctors, working in practices and outpatient clinics, were asked to take part in the study and to report every patient fulfilling the entry criteria (table 1).

On a standardised questionnaire, data on patient history and diagnostic information were collected as well as confirmation that the place of permanent residence was inside the defined study area.

All reported patients who were newly diagnosed as juvenile arthritis in 1995 were called incidence cases and were used in the calculation of the incidence rate. Those patients who were reported in 1995 but had been diagnosed as juvenile arthritis before were called prevalence cases and used in the calculation of the prevalence rate. Thus the point of prevalence was 1 January 1995.

Patients of the incidence group whose complaints continued were scheduled for follow up visits by the reporting doctors up to 9-12 months in order to ensure validated diagnoses at onset. 
Close postal and telephone monitoring was carried out to ensure that the participating doctors returned all the questionnaires and also gave notice if they had not treated any patients fulfilling the inclusion criteria. Multiple reportings of the same patient were detected by comparing initials, date of birth, sex, and residence of all incoming questionnaires.

A pilot study was run in the city of Augsburg to check the feasibility of the main study and its final design. In this pilot study all doctors regardless of their specialism were asked to report retrospectively all cases of juvenile arthritis they had treated within the past 12 months. This pilot study confirmed the results of a survey among patients of the hospital for paediatric rheumatology in GarmischPartenkirchen which had shown that about $83 \%$ of patients with juvenile arthritis were seen by at least one paediatrician, orthopaedist, or rheumatologist.

Healthcare expenses for children and adolescents in Germany are covered by their parents' health insurance until the adolescents themselves, by law, become members of a health insurance. A voluntary health prevention programme for babies, infants, and pre-school children is well established. School children are periodically examined by public health doctors. So every person has the same access to specialist care. To ensure a high number of specialists available we selected an urban study area.

In every town of the study area we briefed all doctors to improve their motivation to contribute to the project and to train them in paediatric rheumatology. They received general information about juvenile arthritis and were informed about the study.

\section{CLASSIFICATION}

In the absence of generally accepted criteria for several forms of juvenile arthritis, diagnoses were made by experts, based on the data given by the reporting doctors, on additional laboratory data, and on the results of our own re-examinations if performed. The diagnosis registered was the one of the patient's first visit to the reporting doctor in 1995 . We applied the EULAR criteria ${ }^{7}$ to classify patients as JCA. In addition to the four "classical" subgroups (systemic, polyarticular, pauciarticular in younger children (= oligoarthritis type I), and pauciarticular in older children (=oligoarthritis type II), we defined a fifth group (unclassified oligoarthritis) for those patients with an oligoarthritis who either did not meet the criteria for any of the two defined types of oligoarthritis (antinuclear antibody positive $v$ HLAB27 positive) or who did fulfil the criteria for both and therefore could not be clearly classified. The oligoarthritis type II subgroup included all cases of juvenile ankylosing spondylitis with active arthritis.

All forms of para-/postinfectious arthritis (PPA), including Lyme arthritis, transient synovitis of the hip ( $\mathrm{SH})$, post-enteric and other forms of post-infectious arthritis were classified as PPA. The only subgroup we differentiated within this group was $\mathrm{SH}$. Criteria for $\mathrm{SH}$ were isolated arthritis of one or two hips, no marked rise in erythrocyte sedimentation rate and leucocytes, no significant fever, possible history of antecedent infection, and exclusion of other relevant diagnoses. Non-PPA and non-JCA forms of arthritis were labelled "other arthritis".

Cases with a definite diagnosis which did not meet the criteria of arthritis were classified as "no arthritis".

Every 10th patient was re-examined by a paediatric rheumatologist from our hospital to validate the diagnosis.

A random sample of 22 practices was taken to review the records of all patients treated during the study period. Thus we ascertained the percentage of patients with juvenile arthritis who were treated by the reporting doctors but were not registered in the study.

\section{STATISTICAL CONSIDERATIONS}

Because we tried to register all cases in the defined region within one year, random sampling error was not a major concern in this study. However, the design of the study suggested that possible biases in reporting and diagnosis should be measured. Their influence on the estimated incidence and prevalence was studied by a correction model.

Reporting bias was estimated from the pilot study and on the basis of the data collected in the review of patients' records. Information on diagnostic bias was gained from the patient validation by re-examination by a paediatric rheumatologist.

A small number of cases which remained unclassified were assigned to the four main diagnostic groups proportionally.

Because the correction model was based on factors estimated from relatively small subsamples a probabilistic sensitivity analysis was performed. This enabled us to show how much the incidence and prevalence results might vary owing to uncertainty of the single correction factors. In a computer based Monte Carlo simulation $^{8} 10000$ estimates were calculated using values for the single correcting factors that were drawn at random and independently of each other from distributions specifying the uncertainty about each factor. The upper and lower $2.5 \%$ centiles of the resulting distribution of an incidence or prevalence rate were defined as borders of uncertainty.

\section{Results}

Of the 766 doctors contacted, 656 doctors $(85.6 \%)$ took part in the study actively. Eighty seven $(11.4 \%)$ only received one and no further mailing because either they declared that they did not treat any patients fulfilling the inclusion criteria in general or because colleagues of theirs in the same practice or clinic were instructed to report their cases. Twenty three doctors $(3 \%)$ refused to participate in the study, even after a request by telephone. 


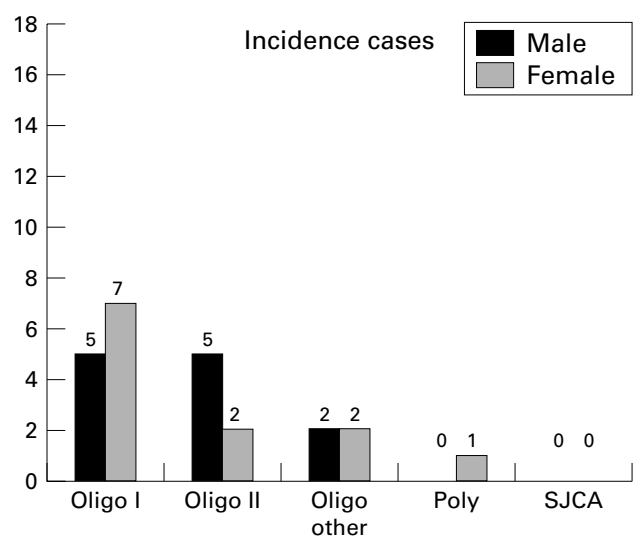

Figure 1 fuvenile chronic arthritis ( $(\mathcal{C} A$ ) subgroups.

REPORTED CASES

Four hundred and fifty seven reported cases fulfilled the inclusion criteria for age, residence, and date of consultation. Of these 457 patients, $294(64.3 \%)$ were diagnosed with PPA, 78 (17.1\%) with JCA, 18 (3.9\%) with other forms of arthritis, and $47(10.3 \%)$ with no arthritis; 20 cases $(4.4 \%)$ remained unclassified. Twenty four of the 78 JCA cases were newly diagnosed in 1995 and thus were considered as incidence cases; the remaining 54 were prevalence cases. Oligoarthritis is the predominant subgroup among patients with JCA of both the incidence and the prevalence group. Twenty three of 24 incidence patients $(96 \%)$ and 42 of 54 prevalence patients $(78 \%)$ showed a pauciarticular disease type (fig 1). In the JCA prevalence group one patient with polyarthritis and two with oligoarthritis fulfilled the Vancouver criteria for psoriatic arthritis. ${ }^{9}$ One patient with prevalent oligoarthritis was diagnosed with inflammatory bowel disease.

Of the 294 PPA cases, 148 were diagnosed with SH. The remaining 146 PPA consisted of inflammatory joint diseases after infections of the gastrointestinal tract, after borrelia and other bacterial or viral infections. Only three of the 148 patients diagnosed with SH and 13 of the 146 remaining patients with PPA were prevalence cases.

Of the 18 cases labelled as "other arthritis", 11 could not be definitely classified as either JCA or PPA, four were diagnosed with

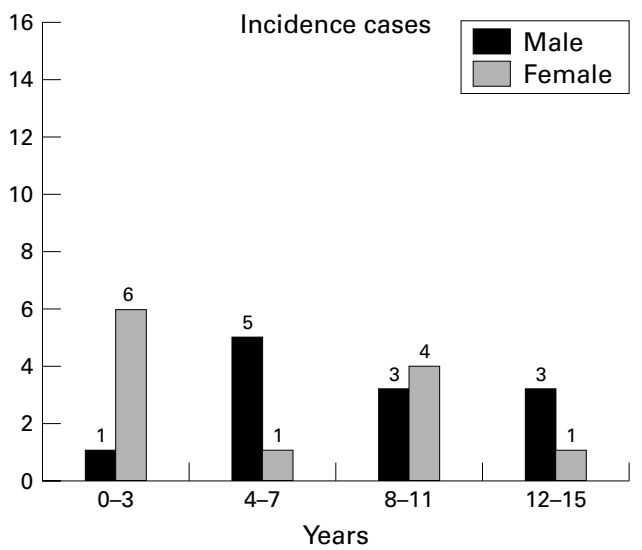

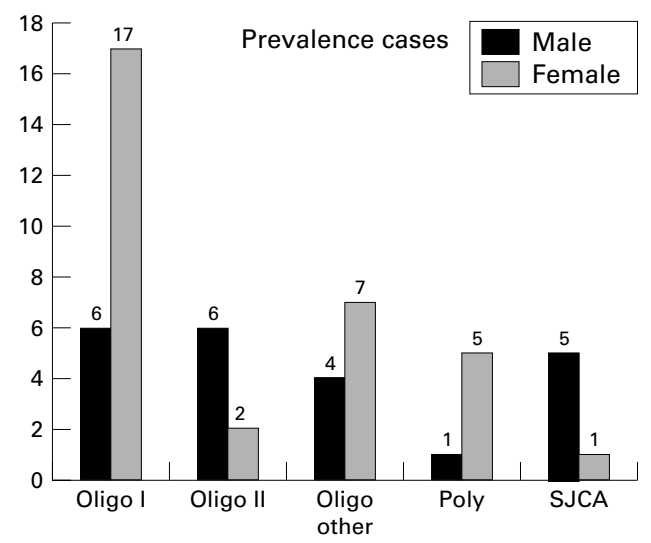

rheumatic fever, two with arthritis in sarcoidosis, and one with arthropathy in gout.

Two patients with connective tissue diseases, one with fibromyalgia, and eight with different forms of vasculitis did not show active arthritis.

AGE AND SEX DISTRIBUTION

The registered age applies to the age at the time of disease onset.

In the JCA prevalence group the ratio of girls to boys was 32:22, in the incidence group both sexes were distributed equally (12:12). The age distribution in the JCA incidence group was almost balanced between those aged under and over 7 (13:11). In the JCA prevalence group there was a predominance of children aged 0-7 years over older ones, with a ratio of 38:16 (fig 2).

The proportion of patients in the age group 12-15 was surprisingly low in both the incidence and the prevalence group. The distribution of the sexes among the older patients was almost equal.

Owing to the small number of prevalent PPA cases we analysed the age and sex distribution only for the incidence cases of this group of diagnoses. The cases with $\mathrm{SH}$ were separated from the rest (figs 3 and 4).

The sex distribution in the patients with $\mathrm{SH}$ was $M: F=105: 40=2 \cdot 6 / 1$. This distribution was almost constant in all ages. The mean age was 6.2 years. These results are consistent with previously published data..$^{10-13}$

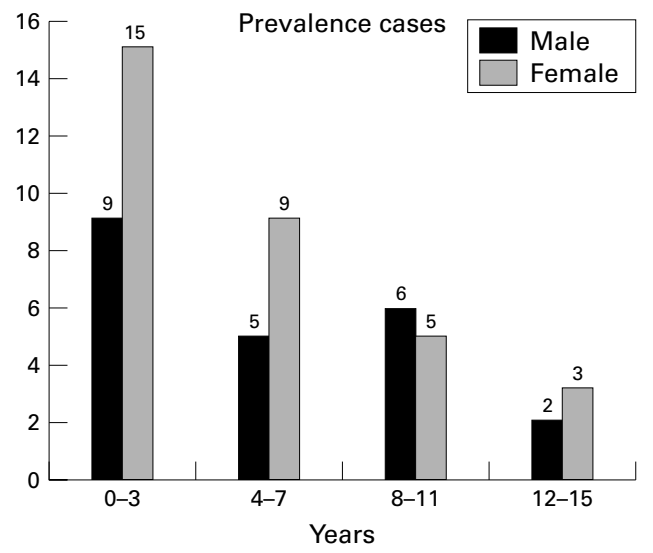

Figure 2 Distribution of age and sex in juvenile chronic arthritis. 


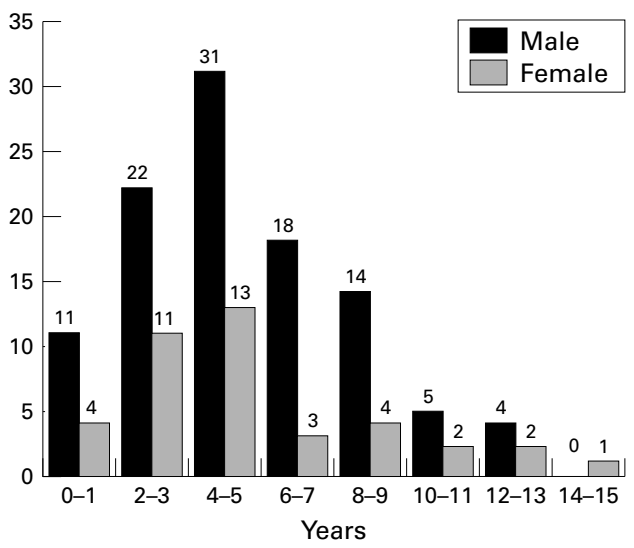

Figure 3 Distribution of age and sex in incident synovitis of the hip.

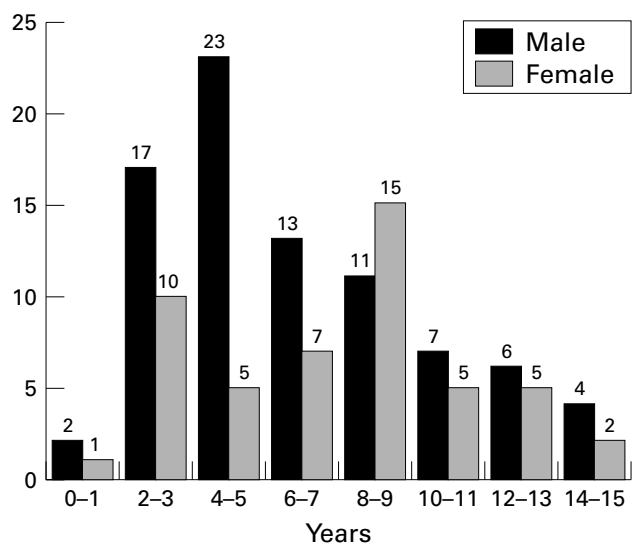

Figure 4 Distribution of age and sex in incident para-/postinfectious arthritis.

Boys were also predominant in the group with other forms of PPA. The ratio boys:girls was $55: 23=2.4: 1$ at age $0-7$ years, but changed to almost $1: 1$ in the $8-15$ year olds. The overall relation boys:girls was $1.7: 1$ for PPA except SH. The mean age was 7.8 years.

CONTROL OF BIAS

Four correction factors (CF) were estimated to control for known sources of bias. The total correction model resulted from multiplying the observed incidence or prevalence by the four single correction factors(table 2).

\section{CF1: Underreporting}

In a review of the records we found an average of five non-reported JCA cases per 100 reported. The rate of non-reported cases was substantially higher in PPA: 162 non-reported cases were discovered per 100 reported.

Table 2 Single correction factors and total correction factor

\begin{tabular}{llllll}
\hline & \multicolumn{2}{l}{$f C A$} & & \multirow{2}{*}{$P P A$} \\
\cline { 2 - 3 } \cline { 5 - 6 } & Incidence & Prevalence & & Incidence & Prevalence \\
\hline Reported cases & 24 & 54 & & 278 & 16 \\
CF1: Underreporting & 1.05 & 1.05 & & 2.62 & 2.62 \\
CF2: Misdiagnosis & 0.85 & 0.85 & & 1.03 & 1.03 \\
CF3: Unclassified & 1.05 & 1.04 & & 1.05 & 1.04 \\
CF4: Selection & 1.21 & 1.21 & & 1.21 & 1.21 \\
Total correction factor & 1.13 & 1.11 & & 3.42 & 3.38 \\
Estimated number of cases (rounded) & 27 & 60 & & 951 & 54 \\
\hline
\end{tabular}

CF2: Diagnostic misclassification

Comparing the diagnoses based on the questionnaires with those made during patient validation, we could estimate the proportion of diagnoses that would have had to be corrected if every patient had been examined by a trained paediatric rheumatologist. Using the method described by Tenenbein, ${ }^{14}$ we estimated the proportion of cases with confirmed JCA and PPA among the 457 patients reported. Not controlling for this bias would have led to a considerable overestimate of JCA and a slight underestimate of PPA.

\section{CF3: Unclassified cases}

The diagnosis of 17 incidence and three prevalence cases remained unclassified. The correction factor was calculated by dividing the number of reported cases by the number of classified cases.

\section{CF4: Selection of reporting doctors}

The results of the pilot study showed that approximately $82.8 \%$ of all patients with juvenile arthritis were treated by a doctor from one of the three selected specialisms. We therefore used a correction factor of $1 / 0.828=1.21$ to control for the bias resulting from restricting our study to paediatricians, orthopaedists, and rheumatologists.

In applying the correction model, we found that the estimated number of JCA cases was close to the number of reported cases: the total correction factor for the incidence of JCA is 1.13 , for the prevalence of JCA it is 1.11 . The difference between the reported and the estimated number of cases with PPA was higher: the total correction factor for the incidence and prevalence of PPA was calculated to be 3.4.

\section{INCIDENCE AND PREVALENCE}

Incidence and prevalence were calculated by relating the reported and estimated numbers of cases to the population at risk in the study area.

The reported annual incidence of JCA in the study area was $6.6(24 / 364939)$, the reported prevalence of JCA was 14.8 (54/364 939) per 100000 people under the age of 16 years. The reported incidence of PPA was 76 (278/ 364 939), and the reported prevalence of PPA was $4.4(16 / 364939)$ per 100000 people under 16 years.

On the basis of the reported data and of the correction model the annual incidence of JCA treated in the study area is estimated to be 7.5 (confidence interval (CI) 5.8 to 12.6) per 100000 , and the prevalence of JCA to be 16.5 (CI 12.5 to 27.5) per 100000.

The annual incidence of PPA treated in the study area is estimated to be 261 (CI 165 to 430) per 100000 and the prevalence of PPA to be 14.8 (CI 9.6 to 25.1) per 100 000. Approximately half of the patients with PPA showed an $\mathrm{SH}$.

The annual incidence of rheumatic fever treated in the study area was estimated to be clearly below 1 per 100000 . 


\section{Discussion}

This is the first prospective study to present data on incidence and prevalence of juvenile arthritis in Germany. Published incidence rates for JCA in other European countries range between 1.3 and 22.6 per 100000 children under the age of 16 years. ${ }^{415}$ The prevalence rates for JCA were estimated to be 10 to 80 per 100000 . Even if the differences between the published data are partly due to differing study methods, there is a clear geographical gradient of JCA frequency with markedly high incidence and prevalence in the Scandinavian countries.

In a retrospective study which was performed by a Berlin study group in the same research programme the incidence for treated JCA was estimated to be 3.5, and the prevalence for treated JCA 20.3 per 100000 children under the age of 16 years. ${ }^{16}$ This may be a slight underestimation of the incidence because retrospective studies tend to underestimate incidence owing to underreporting, especially of mild disease courses. On the other hand, the incidence of JCA in our own study may be slightly overestimated, the prevalence underestimated. Even though prospective studies at primary care level tend to show a higher proportion of oligoarthritis in JCA than hospital based studies, the rate of $96 \%$ and the sex distribution of 1:1 in the incidence group of our study indicate that some cases of PPA may have been misclassified as JCA. Of course it can also be expected that some patients with oligoarthritis at onset will develop polyarthritis in the disease course. This, however, does not explain the low rate of polyarthritis at onset. With all the measures taken for validation of diagnoses we do not think that a relevant number of patients with polyarthritis were misclassified as oligoarthritis.

Prevalence is known to equal approximately the product of incidence and disease duration. ${ }^{17}$ Reported values for the average incidence:prevalence ratio for JCA are $1: 5$ to $1: 6$, meaning that the duration of the disease is about five to six years. The fact that disease duration for JCA is limited by the cut off point at the age of 16 years, given by the diagnostic criteria, must be taken into account.

We found the expected incidence:prevalence ratio for the patients with polyarthritis and systemic disease. (The fact that no incident systemic case was reported can be explained by the small absolute number of cases.) The incidence:prevalence ratio of $1: 1.8$ for the oligoarthritis subgroups, however, markedly exceeds the expected results and confirms the assumption that some misclassified cases of PPA might be in the JCA incidence group.

One possible reason for the underestimation of JCA prevalence is the selection effect, which results from the fact that general practitioners and specialists for internal medicine were not included in the project; doctors of these two specialties preferably treat older children and adolescents. Among the older patients there is a relatively high proportion of prevalence cases, and therefore most of the patients who were not reported owing to the exclusion of the specialists mentioned were prevalence cases. The low number of 11 to 15 year olds among the patients with JCA, as well as those with PPA, supports this hypothesis. Additionally, it seems possible that incidence cases were reported more reliably than prevalence cases.

A small number of patients may have consulted the reporting doctor for the first time in 1995 not informing him or her that the diagnosis was made before 1995 . Thus they might be registered as incidence instead of prevalence cases.

Another possible reason for the underestimation of the JCA prevalence is the exclusion of patients in remission, as arthritis was one of the entry criteria.

Taking these considerations into account, we assume that the true annual incidence for treated JCA in the Berlin area as well as in the Bavarian region is in the lower range of the confidence interval at about 5-6 per 100000 . The treated JCA prevalence is likely to be found in the upper range of the confidence interval at about 25 per 100000 .

Quality controls showed that acute forms of juvenile arthritis were reported less reliably than chronic forms. Therefore the incidence for PPA of 261 per 100000 marks the baseline limit. We think that $250-350$ per 100000 would be a realistic estimate. This incidence is markedly higher than the results of Kunnamo et al in Finland. ${ }^{3} \mathrm{~A}$ higher incidence of PPA and a lower incidence of JCA in Germany compared with Finland might indicate a difference in the pathophysiological background of these two disease groups.

The prevalence of acute PPA is likely to be 15-20 per 100 000. According to these data the mean disease duration of PPA is three weeks.

Because the two studies in GarmischPartenkirchen and in Berlin had similar results, even though different methods were used, we assume that the incidence and prevalence of juvenile arthritis in other parts of Germany are consistent with these data. We therefore estimate the number of incidence and prevalence cases of JCA to be 750-900 and 3600-4350 a year, respectively, in Germany. About 21000 children and adolescents are newly diagnosed with $\mathrm{SH}$ and the same number of patients with other forms of PPA every year in Germany.

The study has shown that thorough quality control measures must be taken to ascertain prospective data on juvenile arthritis. Without these precautions the incidence and prevalence of JCA would have been overestimated and those of PPA would have been substantially underestimated.

We thank all reporting doctors for their valuable contribution to this study, which could not have been performed without their this studp.
help.

This project was supported by the German government in a research programme on the epidemiology of rheumatic diseases (grant Nos 01EF9409 and 01EF9413).

1 Truckenbrodt H. Zur Versorgungsstruktur rheumakranker Kinder und Jugendlicher in Westdeutschland. Z Rheumatol Kinder und Jug

2 Andersson Gäre B, Fasth A. Epidemiology of juvenile chronic arthritis in southwestern Sweden: a 5-year prospective population study. Pediatrics 1992;90:950-8. 
3 Kunnamo I, Kallio P, Pelkonen P. Incidence of arthritis in urban Finnish children. A prospective study. Arthritis Rheum 1986:29:1232-8.

4 Prieur AM, Le Gall E, Karmann F, Edan C, Lasserre O, Goujard J. Epidemiologic survey of juvenile chronic arthritis in France. Clin Exp Rheumatol 1987;5:217-23.

5 Özdogan H, Ksapcopur O, Dede H, Arisoy N, Beceren T, Yurdukal S, et al. Juvenile chronic arthritis in a Turkish population. Clin Exp Rheumatol 1991;9:431-5

6 Kvien TK, Glennas A, Melby K, Granfors K, Andrup O, Karstensen B, et al. Reactive arthritis: incidence, triggering agents and clinical presentation. J Rheumatol 1994;21: 115-22.

7 Wood PF. Special meeting on nomenclature and classification of arthritis in children. In: Munthe E, ed. EULAR monograph series No 3. Basle: EUL AR Publishers, 1978:4750.

8 Doubilet P, Begg CB, Weinstein MC, Braun P, McNeill BJ Probabilistic sensitivity analysis using Monte Carlo simulation: a practical approach. Med Decis Making 1985; simulation:

9 Southwood TR, Petty RE, Malleson PN, Delgado E, Hunt $\mathrm{D}$, Wood B, et al. Psoriatic arthritis in children. Arthritis Rheum 1989;32:1007-13.
10 Briggs RD, Baird KS, Gibson PH. Transient synovitis of the hip joint. J R Coll Surg Edinb 1990;35:48-50.

11 Landin LA Danielsson LG, Wattsgard C. Transient synovitis of the hip. Its incidence, epidemiology and relation to Perthes' disease. J Bone Joint Surg Br 1987;69:238-42.

12 Graf J, Bernd L, Niethard FU, Kaps HP. Die Diagnostik bei der Coxitis fugax, der häufigsten Hüfterkrankung beim Kind. Klin Pädiatr 1991;203:448-51.

13 Vijlbrief AS, Bruijnzeels MA, van der Wouden JC, van Suijlekom-Smit LW. Incidence and management of transient synovitis of the hip: a study in Dutch general practice. Br J Gen Pract 1992;42:426-8.

14 Tenenbein A. A double sampling scheme for estimating from binomial data with misclassifications. Journal of the American Statistical Association 1970;65:1350-61.

15 Moe N, Rygg M. Epidemiology of juvenile chronic arthritis in northern Norway: a ten-year retrospective study. Clin Exp Rheumatol 1998;16:99-101.

16 Kiessling U, Döring E, Listing J, Meincke J, Schöntube M, Strangfeld A, et al. Incidence and prevalence of juvenile ctrangfeld A, et al. Incidence and prevalence of juvenile 1998;25:1837-43.

17 Hennekens $\mathrm{CH}$, Buring JE. Epidemiology in medicine. Boston, Toronto: Little Brown, 1987:65.

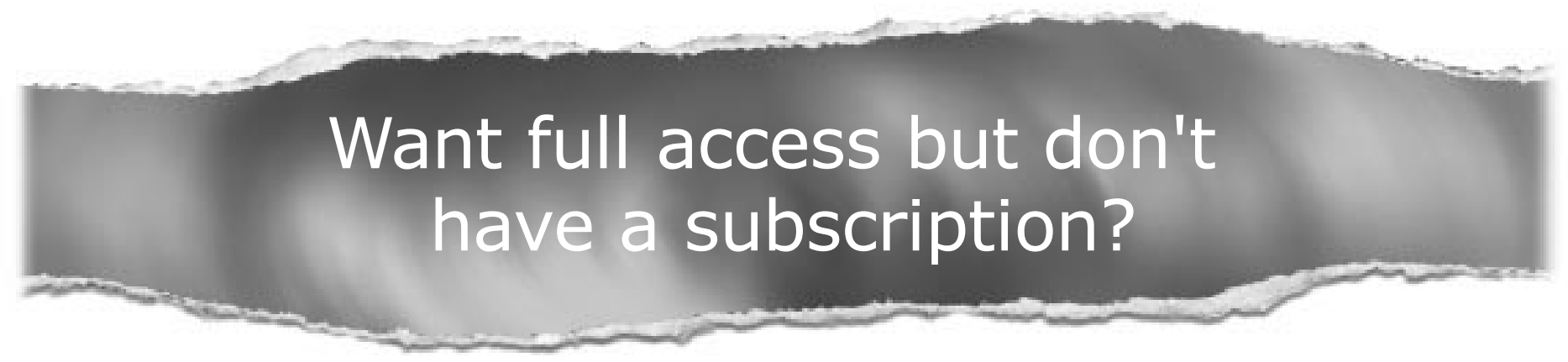

\section{Pay per access}

For just US\$25 you can have instant access to the whole website for 30 days. During this time you will be able to access the full text for all issues (including supplements) available. You will also be able to download and print any relevant pdf files for personal use, and take advantage of all the special features Annals of the Rheumatic Diseases online has to offer.

\section{www.annrheumdis.com}

